\title{
A rare cardiac manifestation of Wegener's granulomatosis
}

We present a case of a 29 -year-old man who was initially presented to our institution with the complaint of fever, cough, facial tenderness and pain. On clinical examination patient had nasal mucosal hypertrophy. His blood examination revealed moderate anemia (hemoglobin: $7.9 \mathrm{~g} / \mathrm{dL}$ ), leucocytosis with neutrophilia (WBC- $13.600 / \mathrm{mm}^{3}$ ) and markedly elevated erythrocyte sedimentation rate (ESR) of $86 \mathrm{~mm} /$ hour and high sensitive C reactive protein (hs-CRP). Paranasal sinus computed tomography (CT) examination revealed bilateral paranasal sinus soft tissue opacification (most marked in maxiller sinuses) with extension of pathology in nasal cavity (Fig. 1). The admission diagnosis was pansinusitis and the patient underwent the treatment with IV ampicillin sulbactam (1 gr four times a day). Despite antibiotic treatment, patient's complaints were not declined and there was an increase in hs-CRP: $258 \mathrm{mg} / \mathrm{L}(<5 \mathrm{U} / \mathrm{L})$ and leukocytosis: $15600 / \mathrm{mm}^{3}$. Patient was admitted to hospital. Patient revealed complaints of cough and dyspnea. His chest X-ray revealed nodular shadow in apical regions, with subsequent chest CT revealing nodular pulmonary lesions in apical segments of both upper lobes. His urine examination and serum creatinine were normal. In the following days, patient revealed complaint of chest pain and shortness of breath. On physical
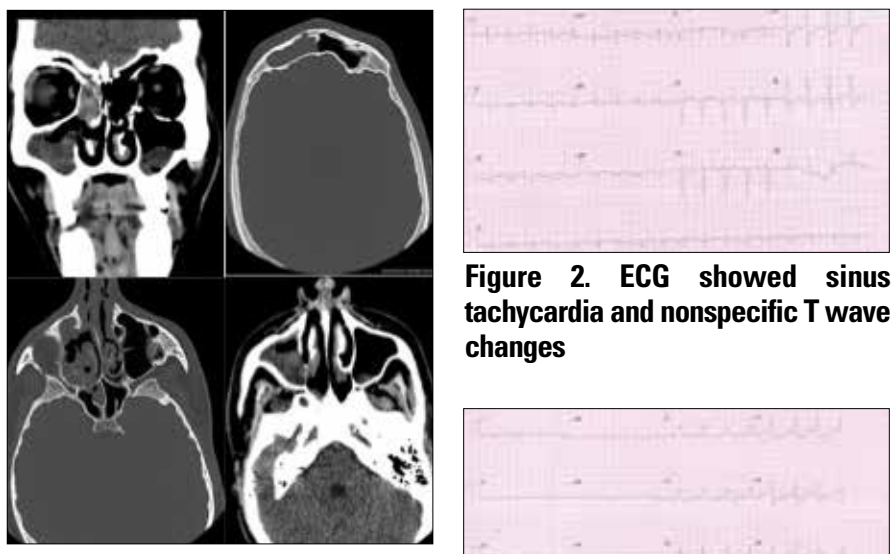

Figure 2. ECG showed sinus tachycardia and nonspecific T wave changes

Figure 1. Paranasal sinus computed tomography (CT) revealed bilateral paranasal sinus soft tissue opacification (most marked in maxiller sinuses) with extension of pathology in nasal cavity examination he was in respiratory distress using accessory muscles and was noted to have 2/6 pansystolic murmur over the apex. The diagnostic workup involved an electrocardiogram (ECG), serial cardiac enzymes. His ECG showed sinus tachycardia and nonspecific T wave changes (Fig. 2). High sensitive troponin was increased a level of $1500 \mathrm{pg} / \mathrm{mL}$ (normal range is $<14$ $\mathrm{pg} / \mathrm{mL}$ ). Two-dimensional echocardiography revealed mild impairment of left ventricular systolic function with ejection fraction $40-45 \%$ and mild spontane echo contrast (SEC) in left ventricle (LV). Based on the complete radiological findings with relevant clinical data a possibility of Wegener's granulomatosis (WG) was thought. The diagnosis was confirmed by histopathological examination of nasal tissue excised on surgery which revealed granulomatous inflammation and presence of antineutrophil cytoplasmic antibody (C-ANCA) in serum. Patient was started pulse dose prednisolone as $1 \mathrm{~g}$ for three days after that $1 \mathrm{mg} / \mathrm{kg}$ on going steroid therapy. In the next days, the patient developed severe dyspne and chest discomfort. On examination, he was found to have low blood pressure $(90 / 60 \mathrm{~mm} \mathrm{Hg})$, pulse rate $110 / \mathrm{min}$, distant heart sounds and crackles throughout his both lung fields. His ECG showed sinus rhythm and complete right bundle branch block (Fig. 3). Transthoracic echocardiography revealed a cardiac mass in LV and severe systolic dysfunction (Video 1-2). Coronary angiography revealed normal coronary arteries. We followed the patient on medical therapy for two weeks to get the remission of WG. After two weeks, control echocardiography was done (Video 3-4). And finally patient went under surgical treatment for the cardiac mass. What is the diagnosis of the patient?

a) Myocarditis related to $W G$ and cardiac sarcoma

b) Myocarditis related to WG and cardiac granuloma

c) Myocarditis related to WG and cardiac thrombus

d) Myocarditis related to antiinflammatory therapy and Cardiac granuloma

Video 1, 2. Transthoracic echocardiography revealed a cardiac mass in LV and severe systolic

Video 3, 4. Control transthoracic 3D echocardiography of cardiac mass. 